\title{
ACONSELHAMENTO TELEFÔNICO PARA JOVENS USUÁRIOS DE CRACKa
}

\author{
Nadia Krubskaya BISCH ${ }^{b}, M$ ariana Canellas BE NCHAYA ${ }^{b}$, Luciana SI G N OR ${ }^{c}, H$ ilda M aria Rodrigues \\ M OLEDA ${ }^{d}, M$ aristela FERIG OLO ${ }^{e}, T$ ânia M oraes Ramos AN D RADE ${ }^{f}, \mathrm{H}$ elena M aria T annhauser BAR ROS ${ }^{9}$
}

\section{RESUM 0}

E ste estudo buscou avaliar a utilização da I nter venção Breve M otivacional (I BM ) em um serviço de aconsel hamento tel efônico, oferecida aos jovens no processo de cessação do consumo de crack. Foi realizado um estudo descritivo, a partir do levantamento dos registros de ligações de usuários no período de janeiro de 2006 a dezembro de 2007. Foram incluídos 40 registros de chamadas de jovens com idades entre 16 e 24 anos que consumiam crack, associado ou não com outras substâncias. Ao final do acompanhamento no sexto mês, $65 \%$ dos jovens haviam parado o consumo de crack. Do total, 57,5\% estavam no estágio de preparação e ação na primeira ligação. As chances para recaída do uso de crack foram maiores nos seguimentos até 30 dias. A partir dos resultados obtidos pode-se sugerir que, a IBM associada ao aconsel hamento tel efônico representa uma alternativa de tratamento importante na cessação do uso de crack.

D escritores: A dolescente. Cocaína crack. M otivação. T el efone. A consel hamento.

\section{RESUMEN}

E ste estudio buscó evaluar el uso de la Intervención B reve M otivacional (IBM ) en un servicio de consejería telefónico que ofrece a los jóvenes en el proceso de cese del consumo de crack. Se realizó un estudio descriptivo, basado en una encuesta de Ilamadas telefónicas a los usuarios durante el período de enero 2006 - diciembre 2007, con 40 registros de llamadas de personas jóvenes de entre 16 y 24 años que consumían crack, asociados o no con otras sustancias. Al final del sexto mes de seguimiento, el 65\% de los jóvenes habían dejado el consumo de crack. Del total, el 57\% estaban en fasede preparación yacción en el primer llamado. L as posibilidades de recaída en el uso de crack fueron mayores en el seguimiento de hasta 30 días. Se sugiere que IBM asociada con la asesoría por teléfono es una opción importante de tratamiento para detener el consumo de crack.

Descriptores: Adolescente Cocaína crack. M otivación. T el éfono. Consejo.

Título: Consejería telefónica para los jóvenes usuarios de crack.

\section{ABST RACT}

This study seeks to evaluate the use of B rief M otivational Intervention in a telephone counseling service offer ed to young people in the process of cessation of crack cocaine use. A descriptive study was conducted, based on a survey of the records of calls from users during the period I anuary 2006 to D ecember 2007. T her esearchers included 40 records from calls of young people aged betw een 16 and 24 years who consumed crack cocaine, associated or not with other substances. At the end of the six-month follow up, $65 \%$ of youths had stopped the consumption of crack cocaine of the total, $57.5 \%$ were in stage of preparation and action on the first call. The chances of relapseto crack cocaine use w erehigher in follow-ups to 30 days. The conclusion suggests that the association of IBM with tel ephone counseling is an important treatment option in cessation of crack cocaine use

D escriptors: A dolescent. Crack cocaine M otivation. Telephone Counseling.

T itle: Telephone counseling for young users of crack cocaine

\footnotetext{
a Extraído da monog rafia de conclusão do Curso de Psicologia da U niversidade L uter ana do Brasil apresentada em 2008.

b Psicóloga, M estranda pelo Programa de Pós G raduação em Ciências da Saúde da U niversidade Federal de Ciências da Saúde de Porto Alegre (UFCSPA), Supervisora do Serviço Nacional de Orientações e Informações Sobre a Prevenção do U so Indevido de Drogas (VIVAVOZ), Porto Alegre, Rio G rande do Sul, Brasil.

c M estre em Ciências da Saúde, Supervisora do VIVAVOZ, Porto Alegre, Rio G rande do Sul, Brasil.

- Enfer meira, M estranda pelo Programa de Pós Graduação em Ciências da Saúde da U FCSPA, Supervisora do VIVAVOZ, Porto A legre, RS, Brasil.

e D outora em Ciências M édicas, Coordenadora do VIVAVOZ, Porto Alegre, Rio Grande do Sul, Brasil.

f M estre em Psicologia Clínica, Especialista em Dependência Química e Terapia Cognitivo-Comportamental, Professora do Curso de Psicologia da Universidade Luterana do Brasil (U LBRA), Canoas, Rio Grande do Sul, Brasil.

a Ṕ́s-D outora em N europsicofar macologia, Professora T itular do D epartamento de F ar macologia da U F CSPA, Coordenadora do VIVAVOZ, Porto Alegre, Rio Grande do Sul, Brasil.
} 


\section{INT RODUÇÃO}

Os levantamentos epidemiológicos de âmbito nacional sobre uso de drogas, realizados pelo Centro Brasileiro de Informações sobre Drogas Psicotrópicas (CEBRID), têm demonstrado o preocupante uso de crack no Brasil ${ }^{(1,2)}$. Q uando comparado 0 consumo desta substância entre 2001 e 2005, o II Levantamento Domiciliar realizado nas 108 maiores cidades do país mostrou um aumento no consumo de $0,5 \%$ para $1,1 \%$. 0 estudo verificou, ainda, que o uso na vida de crack é maior entre os homens em todas as faixas etárias, sendo que entre homens de 12 a 17 anos e de 18 a 24 anos o uso na vida foi de $2,9 \%$ (1).

Os prejuízos que o consumo de crack acarre ta nos jovens levam a consequências de difícil reversão, como a interrupção do desenvolvimento da personalidade, resultando em deficiências futuras do funcionamento do indivíduo. T al consumo afeta, ainda, o desenvolvimento das funções cognitivas e sociais do sujeito, bem como o estabelecimento de relações interpessoais $s^{(3)}$.

$\mathrm{N}$ a tentativa de atenuar tais consequências, a Entrevista M otivacional (EM) surge como alternativa de tratamento. Esta apresenta características positivas no que se refere à intervenção para jovens dependentes de crack e outras drogas, tais como não-confrontação, fortalecimento da autoeficácia e planejamento de estratégias a curto prazo. A lém disso, trabal ha diretamente com a resolução da ambivalência, característica comum nesta fase do desenvolvimento, minimizando a resistência para mudança comportamental (4).

Baseada na EM, a Intervenção Breve M otivacional (IBM ) é uma técnica de curta duração que aliada às intervenções tel efônicas pode ser uma alternativa de tratamento para usuários de substâncias psicoativas, com a vantagem de poder ser aplicada não só por especialistas, mas por qualquer profissional capacitado e treinado. E ste tipo de intervenção vem sendo utilizada como estratégia de prevenção da recaída, otimização do aconsel hamento oferecido por profissionais de saúde ${ }^{(5-7)}$ e também como uma modalidade de tratamento(8).

0 aconsel hamento tel efônico apresenta vantagens, como o fato do cliente obter um serviço na sua própria residência. Indivíduos com limitações físicas ou que moram em regiões onde não há acesso a tratamento, ou que são relutantes em participar de intervenções face-a-face também são bene- ficiados com esta modalidade ${ }^{(9)}$. A IBM e 0 apoio tel efônico podem ser alternativas importantes na mudança comportamental do usuário, entretanto são necessárias pesquisas sobre a efetividade e utilização destas ferramentas associadas.

N o Brasil, o Serviço Nacional de Orientações e Informações Sobre a Prevenção do U so Indevido de Drogas (VIVAVOZ) é a primeira central telefônica aberta à população em geral que oferece orientações e informações a respeito de substâncias psicoativas, sua ação no organismo, prevenção ao uso e recursos disponíveis na comunidade; além de oferecer aconselhamento telefônico personalizado, baseado na IBM , aos usuários de drogas e familiares.

F rente às consequências nocivas do consumo de crack para o desenvolvimento dos jovens, além do déficit em pesquisas sobre a efetividade da IBM por telefone aplicada a esta população, o presente estudo buscou avaliar a utilização desta intervenção, em um serviço de aconsel hamento telefônico, oferecida aos jovens no processo de cessação do consumo de crack.

\section{MÉTODO}

Realizou-se um estudo descritivo retrospectivo com abordagem quantitativa no período de janeiro de 2006 a dezembro de 2007. 0 estudo fez parte da monografia de conclusão de curso intitulada "A U tilização da Intervenção Breve M otivacional em A dolescentes e A dultos J ovens U suários de Crack A tendidos em um Serviço de A consel hamento Telefônico"(10). Foram incluídos 40 registros de chamadas de jovens que ligaram para 0 VIVAVOZ, das cinco regiões brasileiras, com idades entre 16 e 24 anos, usuários de crack. Os participantes foram incluídos no estudo por meio de um processo de consentimento informado, realizado verbalmente, com a aceitação tácita da participação dos jovens após exposição dos objetivos e da finalidade do projeto. A aceitação oral foi devidamente registrada nos protocolos informatizados. 0 consentimento foi obtido por etapas (rolling consent) visto que os jovens voltaram a ligar seis vezes para acompanhamento ou forneceram número telefônico com o qual foi possível fazer contatos próativos (o serviço telefona para o cliente). 0 presente estudo foi encaminhado e aprovado pelo Comitê de É tica e Pesquisa da U niversidade Federal de Ciências da Saúde de Porto A legre (U F CSPA), pro- 
cesso número 200/ 2006, conforme parecer número 325/ 2007.

As chamadas tel efônicas analisadas foram correspondentes à primeira ligação e as ligações de acompanhamento, baseadas em entrevista estruturada telefônica com duração média de $40 \mathrm{mi}$ nutos. A entrevista incluiu os seguintes instrumentos:

a) Protocolo G eral de A tendimento - contendo informações como sexo, idade, estado marital, profissão, renda familiar, escolaridade, cidade da qual foi realizada a chamada, hora e duração da chamada e dados sobre a classificação da pergunta, incluindo busca por centros de tratamento, informações sobre drogas, material informativo, outras e retorno, além do tipo de droga utilizada;

b) Questionário para Avaliação do Consumo de Crack - caracterizava a quantidade, frequência e padrão de consumo. Foram investigadas as categorias: uso no mês, frequência no último mês e; quantidade usada no mesmo dia(11). Para isto, foi utilizado um questionário elaborado pela Organização M undial da Saúde (OM S) o qual tem sido amplamente utilizado no Brasil(2);

c) Questionário para Avaliação da D ependência de Crack - utilizado para estimar dependência, sendo baseado em 6 dos 9 itens existentes no $M$ anual Diagnóstico e E statístico de T ranstornos $M$ entais, 4a edição, texto revisado (DSM -IV-T R). Os respondentes são definidos como dependentes de crack, caso respondam afirmativamente a pelo menos 2 critérios do questionário(11);

d) Contemplation $L$ adder - utilizada para identificar a prontidão para mudança do jovem ${ }^{(12)}$. Contém 11 pontos contínuos representados por 10 degraus de uma escada. N estes degraus encontramse frases afirmativas que descrevem estágios de prontidão para parar de fumar crack (pré-contemplação, contemplação, preparação e ação). Foi adaptada para ser utilizada nesta população;

e) Intervenção Breve M otivacional - adaptada para 0 aconselhamento oferecido pelo Serviço; enfatiza a identificação do estágio motivacional de mudança, no qual se encontra 0 cliente e 0 desenvolvimento de planos para mudança comportamental apropriado a cada indivíduo. 0 aconselhamento inclui técnicas para aumentar a motivação para mudança; a promoção da auto-eficácia; a identificação de situações de risco e estratégias de enfrentamento para estas; planejamento da data de parada, além do envio de material de autoajuda(13); f) Ligações de Seguimento - realizadas para acompanhar os jovens durante o processo de redução e ou cessação do consumo. A ssim, objetivou-se verificar aquel es jovens modificaram seu hábito de fumar crack, parando ou reduzindo, os que não mudaram este hábito e ainda, aqueles que recaíram. $\mathrm{Na}$ ligação de seguimento foram anotadas respostas adicionais como: quantidade de dias abstinentes, recaídas, uso de alguma medicação e ou tratamento durante o período de seguimento, realizado em 24 horas, três dias, sete dias, um, dois, três e seis meses. As ligações foram relacionadas ao protocolo de primeira ligação.

Os atendimentos aos clientes do VIVAVOZ são realizados por consultores/ estagiários, acadêmicos dos cursos da área da saúde, previamente treinados e orientados por uma equipe de supervisores, mestrandos e doutorandos do Programa de Pós-G raduação em Ciências da Saúde da U niversidade Federal de Ciências da Saúde de Porto A legre (UFCSPA). 0 aconsel hamento, para os usuários de drogas, ocorre de for ma padrão, utilizandose um fluxograma de orientação específico do Serviço.

No momento em que o cliente telefonava para o Serviç̧o, ele respondia ao Protocolo Geral de A tendimento a fim de se obter dados gerais de caracterização, assim como classificação da pergunta e tipo de droga. Quando identificado como usuário de crack, associado ou não ao uso de outras drogas, era oferecido auxílio para cessar o consumo. Então, o consultor solicitava seu consentimento para uso de dados em pesquisa e iniciava a intervenção e aplicação dos questionários.

0 aconsel hamento, baseado nos princípios da IBM , visava auxiliar o jovem a se movimentar entre os estágios motivacionais de mudança a fim de se motivar para a cessação do consumo. A pós aplicação dos instrumentos, o consultor buscava promover 0 aumento da motivação para o processo de tomada de decisão utilizando técnicas como escuta reflexiva; incentivo às afirmações automotivacionais por parte cliente e; balança decisória. A partir disto, o consultor real izava o planejamento da mudança e prevenção de recaída, reforçando a autoeficácia do cliente e marcava a data da cessação do consumo, agendando as ligações de seguimento. $N$ estas ligações foi investigado, a partir do relato dos indivíduos, se a IBM conduziu a cessação do consumo de crack. 
Os dados levantados for am registrados em um software específico do Serviço e posteriormente resgatados em planilha do M icrosoft E xcel. Com isto, foi montado um banco de dados, para a análise no programa Statistical Package for the Social Sciences (SPSS). Para as análises univariadas as variáveis foram descritas por números absolutos e percentuais. As análises bivariadas foram realizadas por meio do teste Qui-quadrado. Ainda, foi realizada uma Curva de Kaplan M eier Survival para mostrar a recaída dos usuários no período de 180 dias. Foi considerado estatisticamente significativo $p<0,05$. Os programas utilizados para as análises foram SPSS 12.0 e E pl nfo 3.3.2.

\section{RESULT AD OS}

Foram incluídos no estudo 40 jovens atendidos no serviço de aconsel hamento tel efônico que receberam IBM por um período de 6 meses. $0 \mathrm{~b}$ servou-se que ao final do acompanhamento, $65 \%$ dos usuários pararam o consumo de crack e $35 \%$ não pararam o consumo.

As características de gênero da amostra apontam que $67,5 \%$ dos jovens eram do sexo masculino e $32,5 \%$ do sexo feminino. Os dados da comparação entre as variáveis sociodemog ráficas e a cessação do consumo de crack podem ser observados na Tabela 1.

T abela 1 - Características sociodemográficas e a situação da cessação do consumo de crack ao final do acompanhamento de seis meses dos usuários de 16 a 24 anos atendidos em um serviço de aconsel hamento tel efônico. Brasil, jan. 2006 a dez. 2007.

\begin{tabular}{|c|c|c|c|c|c|}
\hline \multirow{2}{*}{ Variáveis } & \multicolumn{2}{|c|}{ Não Parou } & \multicolumn{2}{|c|}{ Parou } & \multirow{2}{*}{ Valor de $p$} \\
\hline & $\mathbf{n}$ & $\%$ & $n$ & $\%$ & \\
\hline \multicolumn{6}{|l|}{ Sexo } \\
\hline M asculino & 9 & 22,5 & 18 & 45,0 & \multirow{2}{*}{1,000} \\
\hline Feminino & 5 & 12,5 & 8 & 20,0 & \\
\hline \multicolumn{6}{|l|}{ Idade } \\
\hline 16 a 20 anos & 4 & 10,0 & 13 & 32,5 & \multirow{2}{*}{0,315} \\
\hline 21 a 24 anos & 10 & 25,0 & 13 & 32,5 & \\
\hline \multicolumn{6}{|l|}{ Escolaridade } \\
\hline A nalfabeto & - & - & 1 & 2,6 & \multirow{5}{*}{0,554} \\
\hline Ensino F undamental & 8 & 20,5 & 8 & 20,5 & \\
\hline Ensino M édio & 5 & 12,8 & 12 & 30,8 & \\
\hline Técnico & - & - & 1 & 2,6 & \\
\hline Ensino Superior & 1 & 2,6 & 3 & 7,7 & \\
\hline \multicolumn{6}{|l|}{ E stado civil } \\
\hline Solteiro & 11 & 27,5 & 20 & 50,0 & \multirow{2}{*}{0,617} \\
\hline Casado & 3 & 7,5 & 6 & 15,0 & \\
\hline \multicolumn{6}{|l|}{ R enda familiar } \\
\hline 1 a 5 salários mínimos & 12 & 30,0 & 18 & 45,0 & \multirow{3}{*}{0,415} \\
\hline 5 a 10 salários mínimos & 2 & 5,0 & 6 & 15,0 & \\
\hline $\mathrm{N}$ ão respondeu & - & - & 2 & 5,0 & \\
\hline \multicolumn{6}{|l|}{ Profissão } \\
\hline Estudante & 2 & 5,0 & 7 & 17,5 & \multirow{3}{*}{0,556} \\
\hline Trabalha & 7 & 17,5 & 13 & 32,5 & \\
\hline D esempregado & 5 & 12,5 & 6 & 15,0 & \\
\hline \multicolumn{6}{|l|}{ Região do país } \\
\hline Norte & 1 & 2,6 & 1 & 2,6 & \multirow{5}{*}{0,981} \\
\hline N ordeste & 2 & 5,3 & 3 & 7,9 & \\
\hline Centro- 0 este & 1 & 2,6 & 2 & 5,3 & \\
\hline Sudeste & 3 & 7,9 & 7 & 18,4 & \\
\hline Sul & 7 & 18,4 & 11 & 28,9 & \\
\hline
\end{tabular}

* T este do qui-quadrado para comparação das características sociodemográficas e a situação ao final do acompanhamento de seis meses. 
A maioria dos jovens usuários de crack já havia buscado algum tipo de tratamento anterior a ligação para o Serviço (62,5\%). N o que se refere às características do consumo de crack, a maioria dos jovens referiu usar a dois anos ou mais ( $55 \%)$, havia feito uso no último mês $(77,5 \%)$, consumia a substância diariamente (57,5\%) e na quantidade que variava de 1 a 14 pedras por dia (65\%). Quanto ao estágio de motivação para mudança, a maioria dos jovens estava preparando-se para modificar o hábito de fumar crack e fazendo algo para modificá-
Io (87,5\%). Os dados referentes à busca de tratamento prévio, características de consumo de substâncias e estágio motivacional relatados na primeira ligação comparados com a situação de cessação do consumo são apresentados na T abela 2.

$\mathrm{N}$ as análises bivariadas relacionadas ao consumo no último mês, observou-se que todos aqueles abstinentes na primeira ligação, permaneceram assim ao final dos seis meses, mostrando uma diferença estatisticamente significativa $(p=0,016)$ (T abela 2).

T abela 2 - Características de tratamento prévio, consumo de drogas e estágio de mudança na primeira ligação e situação de cessação do crack ao final do acompanhamento de seis meses dos usuários de 16 a 24 anos atendidos em um serviço de aconselhamento telefônico. Brasil, jan. 2006 a dez. de 2007.

\begin{tabular}{|c|c|c|c|c|c|}
\hline \multirow{2}{*}{ Variáveis } & \multicolumn{2}{|c|}{ Não Parou } & \multicolumn{2}{|c|}{ Parou } & \multirow{2}{*}{ Valor de $p^{\star}$} \\
\hline & $\mathbf{n}$ & $\%$ & $\mathbf{n}$ & $\%$ & \\
\hline \multicolumn{6}{|l|}{$\mathrm{T}$ ratamento anterior } \\
\hline Sim & 9 & 22,5 & 16 & 40,0 & \multirow{2}{*}{1,000} \\
\hline Não & 5 & 12,5 & 10 & 25,0 & \\
\hline \multicolumn{6}{|l|}{ Tempo de uso } \\
\hline 2 anos ou mais de uso & 9 & 22,5 & 13 & 32,5 & \multirow{3}{*}{0,627} \\
\hline De 6 meses a 2 anos & 4 & 10,0 & 9 & 22,5 & \\
\hline A té 6 meses & 1 & 2,5 & 4 & 10,0 & \\
\hline \multicolumn{6}{|l|}{ Consumo no último mês } \\
\hline Sim & 14 & 35,0 & 17 & 42,5 & \multirow{2}{*}{$0,012^{\dagger}$} \\
\hline Não & - & - & 9 & 22,5 & \\
\hline \multicolumn{6}{|l|}{ Frequência de consumo } \\
\hline Diariamente & 7 & 17,5 & 16 & 40,0 & \multirow{3}{*}{0,338} \\
\hline Semanal mente ou menos & 6 & 15,0 & 6 & 15,0 & \\
\hline Não usou no último mês & 1 & 2,5 & 4 & 10,0 & \\
\hline \multicolumn{6}{|l|}{ Q uantidade de crack no mesmo dia } \\
\hline M ais de 31 pedras & 2 & 5,0 & - & - & \multirow{4}{*}{0,089} \\
\hline De 15 a 30 pedras & 5 & 12,5 & 5 & 12,5 & \\
\hline De 1 a 14 pedras & 7 & 17,5 & 19 & 47,5 & \\
\hline N enhuma pedra & - & - & 2 & 5,0 & \\
\hline \multicolumn{6}{|l|}{ D rogas associadas } \\
\hline Sim & 12 & 30,0 & 24 & 60,0 & \multirow{2}{*}{0,602} \\
\hline Não & 2 & 5,0 & 2 & 5,0 & \\
\hline \multicolumn{6}{|l|}{ E stágio de mudança } \\
\hline Pré-contemplação e contemplação & 2 & 5,0 & 3 & 7,5 & \multirow{3}{*}{1,000} \\
\hline N a primeira ligação & & & & & \\
\hline Preparação e ação & 12 & 30,0 & 23 & 57,5 & \\
\hline
\end{tabular}

* Teste do qui-quadrado para comparação das características de tratamento prévio, consumo de drogas e estágio de mudança na primeira ligação e da situação ao final do acompanhamento de seis meses. $+\mathrm{p}<0,05$.

0 uso do crack associado a outras drogas, lícitas ou ilícitas, foi relatado por $90 \%$ dos participantes. As substâncias associadas a este consumo mais relatadas pelos jovens foram maconha, álcool, tabaco e solventes. A T abela 3 descreve a comparação entre a utilização destas drogas com a situação de abstinência no sexto mês, a seguir. 
T abela 3 - D rogas associadas ao consumo de crack e a situação de cessação do consumo de crack ao final do acompanhamento de seis meses dos usuários de 16 a 24 anos atendidos em um serviço de aconsel hamento tel efônico. Brasil, jan. 2006 a dez. 2007.

\begin{tabular}{|c|c|c|c|c|c|}
\hline \multirow{2}{*}{ Variáveis } & \multicolumn{2}{|c|}{ Não Parou } & \multicolumn{2}{|c|}{ Parou } & \multirow{2}{*}{ Valor de $p^{*}$} \\
\hline & $n$ & $\%$ & $n$ & $\%$ & \\
\hline \multicolumn{6}{|l|}{ M aconha } \\
\hline Sim & 9 & 22,5 & 23 & 57,5 & \multirow{2}{*}{0,102} \\
\hline Não & 5 & 12,5 & 3 & 7,5 & \\
\hline \multicolumn{6}{|l|}{ Álcool } \\
\hline Sim & 9 & 22,5 & 15 & 37,5 & \multirow{2}{*}{0,746} \\
\hline N ão & 5 & 12,5 & 11 & 27,5 & \\
\hline \multicolumn{6}{|l|}{ T abaco } \\
\hline Sim & 10 & 25,0 & 13 & 32,5 & \multirow[b]{2}{*}{0,315} \\
\hline Não & 4 & 10,0 & 13 & 32,5 & \\
\hline \multicolumn{6}{|l|}{ Solvente } \\
\hline Sim & 1 & 2,5 & 6 & 15,0 & \multirow[t]{2}{*}{0,387} \\
\hline Não & 13 & 32,5 & 20 & 50,0 & \\
\hline
\end{tabular}

* Teste do qui-quadrado para comparação do consumo de drogas associadas e a situação de cessação ao final do acompanhamento de seis meses.

As chances para recaída do uso de crack foram maiores nos seguimentos até 30 dias, conforme observado pela curva Kaplan M eier de sobrevida (G ráfico 1). N o entanto, apesar da recaída nos primeiros 30 dias, a grande maioria se encontrava nos estágios motivacionais mais elevados de prontidão para mudança, que correspondem à preparação e ação.

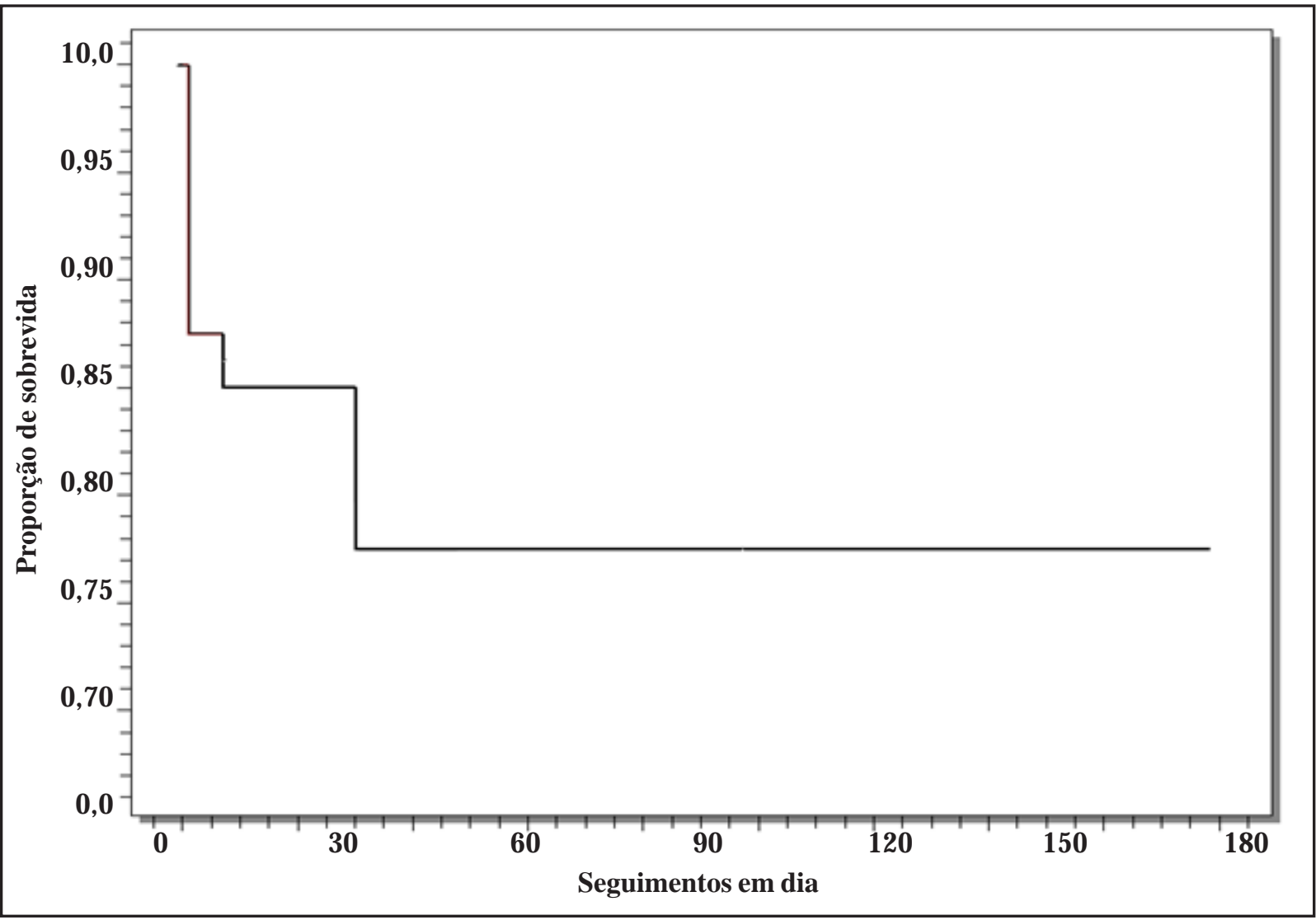

G ráfico 1 - Curva de Kaplan M eier para recaída do uso de crack no período de acompanhamento. Brasil, jan. 2006 a dez. 2007. 


\section{DISCUSSÃO}

Estudos sobre alternativas de tratamento para jovens usuários de crack são de extrema relevância frente ao aumento do uso de drogas por esta população e as dificuldades de acesso ao tratamento na rede pública de saúde.

Ao final do sexto mês de acompanhamento, $65 \%$ dos jovens que aderiram ao estudo haviam parado com o consumo de crack. Estes dados corroboram com a literatura, visto que utilização da IBM tem se mostrado eficaz na abordagem de comportamentos aditivos como de tabaco, álcool e outras drogas ilícitas ${ }^{(7,8,14)}$.

D a mesma forma, o telefone tornou-se uma alternativa para intervenções de mudança comportamental e serviços de saúde, reduzindo custos e possibilitando um gerenciamento mais eficiente da demanda de pacientes em serviços de atenção básica à saúde ${ }^{(15)}$. A s linhas tel efônicas de ajuda têm apresentado vantagens, não somente pelo fato de que atendem um grande número de indivíduos, mas porque é mais acessível àqueles que não têm acesso ou interesse em outros tratamentos como os de face a face ou farmacológicas ${ }^{(9)}$.

No presente estudo, observou-se que os jovens que utilizavam mais de 31 pedras diariamente (5\%) não manifestaram abstinência ao final do acompanhamento, o que pode estar relacionada à maior dificuldade de cessar o consumo da substância quando esta é consumida em quantidades elevadas. Verificou-se também que $32,5 \%$ da amostra utilizou crack por 2 anos ou mais, sendo que $47,5 \%$ utilizava 1 à 14 pedras diárias, o que reflete aspectos sobre peculiaridades farmacocinéticas do crack, como o rápido início de ação e a pronta resolução dos efeitos. I sto leva o usuário à repetição do uso da droga em curtos interval os de tempo o que caracteriza a el evada potencialidade para o desenvolvimento da dependência.

Todos os participantes preencheram os critérios diagnósticos para dependência do crack, o que reforça a infor mação sobre a potencialidade dos efeitos da droga para o desenvolvimento da dependência. A euforia desencadeada pelo uso reforça e motiva o desejo por um novo episódio de consumo, porém quanto mais rápido o início da ação, maior será a intensidade e menor sua duração, e assim, maior será a chance do indivíduo evoluir para situações de uso nocivo e dependência ${ }^{(16)}$. Por essas razões, oferecer um acompanhamento, a esta popula- ção, gratuito e de fácil acesso, que auxilie no processo de cessação do consumo torna-se necessário, visto que quanto mais precoce o início do uso da droga, maiores serão as consequências para a vida do jovem.

No que se refere ao consumo de crack e associação a outros tipos de drogas, $90 \%$ da amostra referiram utilizar drogas associadas. E ste dado corrobora com estudos que referem que o usuário de crack, com frequência utiliza outras substâncias, em especial o álcool, o tabaco e a maconha, sendo a maconha a primeira droga ilícita utilizada ${ }^{(17)}$.

D os jovens que cessaram o consumo de crack no final do sexto mês, $40 \%$ já havia buscado outras formas de tratamento antes da primeira ligação. A literatura aponta que a busca por tratamento parece ser mais precoce entre os usuários de crack, em comparação aos de cocaína intranasal ${ }^{(17)}$, o quetambém pode estar relacionado às consequências decorrentes desta droga ${ }^{(18)}$.

Com relação à cessação do uso da droga, a maioria dos participantes encontrava-se abstinentes ao final do acompanhamento, destes $57,5 \%$ estavam nos estágios de preparação e ação na primeira ligação após a intervenção. Conforme o modelo transteórico ${ }^{(19)}$ a prontidão para mudança consiste na premissa de que a mudança comportamental é um processo e que as pessoas têm diversos níveis de motivação nos quais o indivíduo transita. N o estágio de preparação, o indivíduo já tem a intenção concreta de modificação e mudança, mas ainda é necessário um planejamento de ação. $\mathrm{Na}$ ação, o sujeito está decidido a mudar e já está fazendo alguma coisa para sua mudança comportamental ${ }^{(19)}$.

Durante o período de acompanhamento, as chances de recaída foram maiores nos primeiros 30 dias. Entende-se por recaída a volta ao padrão de consumo anterior, o qual faz parte do processo de mudança e é muitas vezes o modo como a pessoa aprende e recomece o tratamento de forma mais consciente $\mathrm{e}^{(14,19)}$

0 papel do profissional na IBM pauta-se em cinco princípios, são eles: expressar empatia, desenvolver a discrepância, evitar a confrontação, fluir com a resistência e estimular a autoeficácia(20). Para a abordagem com jovens, estes cinco princípios não precisam ser adaptados, mas é necessária maior atenção quanto às peculiaridades da faixa etária devido à importância da relação profissional e cliente. 
As estratégias utilizadas per mitem avaliar 0 estágio de prontidão para mudança, aconsel har 0 cliente conforme 0 estágio de mudança identificado e trabalhar a ambivalência para que então se obtenha a abstinência ${ }^{(20)}$. Desta forma, os dados obtidos demonstram que a IBM por telefone auxiliou grande parte dos jovens estudados a cessarem o consumo do crack durante o período de seguimento, mantendo-os nos estágios mais elevados de motivação.

Pode-se verificar também, em estudo(14) que visava estimular a mudança comportamental com uso da entrevista motivacional em jovens infratores, que após a reavaliação destes obteve-se um aumento do número de dias de abstinências de maconha e álcool, a diminuição da quantidade de uso de álcool, tabaco e maconha e a abstinência de cocaína, crack e solventes.

\section{CONCLUSÕES}

Os resultados mostram que a IBM oferecida por meio de aconselhamento telefônico conduziram os jovens a abstinência do uso de crack durante o período de acompanhamento proposto neste estudo. 0 estudo sugere que a IBM associada ao aconsel hamento tel efônico representa uma alternativa de tratamento importante, principalmente nos casos em que há dificuldade de acesso e dificuldade financeira.

Pesquisas sobre o tema com amostras maiores precisam ser realizadas para que se possa investigar a eficácia da IBM por tel efone em jovens usuários de crack, complementado resultados encontrados neste estudo e possibilitando maior entendimento desta modalidade de atendimento.

\section{REFERÊ NCIAS}

1 Carlini EA, G alduróz JCF, N oto AR, Fonseca AM, Carlini CM . II levantamento domiciliar sobre o uso de drogas psicotrópicas no Brasil: estudo envolvendo as 108 maiores cidades do país. São Paulo: Centro Brasileiro de Infor mações sobre D rogas Psicotrópicas, U niver sidade F ederal de São Paulo; 2007.

2 G alduróz JCF, N oto AR, Fonseca A M , Carlini EA. V levantamento sobre o consumo de drogas psicotrópicas entre estudantes do ensino fundamental e médio da rede pública de ensino nas 27 capitais brasileiras. São Paulo: Centro Brasileiro de Informações sobre Drogas Psicotrópicas, Universidade Federal de São Paulo; 2004.
3 M inistério da Justiça (BR), Secretaria Nacional de Políticas sobre D rogas. Conversando sobre cocaína e crack. Brasília (DF); 1999.

4 Baer J S, Peterson PL. M otivational interviewing with adolescents and young adults. In: M iller RW, Rollnick $S$, editors. M otivational interviewing: preparing people for change. 2nd ed. N ew York: G uilford; 2002. p. 320-32.

5 Chaves E C, Oyama SM R. A consel hamento tel efônico para cessação do tabagismo. Rev G aúcha $\mathrm{E}$ nferm. 2008;29(4):513-9.

6 M azoni CG, Bisch NK, Freese L, Ferigolo M, Barros H M T. A consel hamento tel efônico reativo para cessação do consumo do tabaco: relato de caso. Aletheia. 2006;24:137-48.

$7 \mathrm{M} \mathrm{cC}$ ambridge J, Strang J. The efficacy of singlesession motivational interviewing in reducing drug consumption and perceptions of drug-related risk and harm among young people: results from a multi-site cluster randomized trial. A ddiction. 2004;99:39-52.

8 Rubak S, Sandbak A, L auritzen T, Borch-J ohnsen K, Christensen $B$. An education and training course in motivational interviewing influence: G Ps' professional behaviour. Br J G en Pract. 2006;56:429-36.

9 Galanti R, A hnve S, Gilljam H. Factors related to abstinence in a telephone helpline for smoking cessation. Eur J Public Health. 2004;14(3):306-10.

10 Bisch NK, Andrade T, Ferigolo M . A utilização da Intervenção Breve M otivacional em adolescentes e adultos jovens usuários de crack atendidos em um serviço de aconsel hamento telefônico [ monog rafia] . Canoas: Curso de Psicologia, U niversidade L uterana do Brasil; 2008.

11 Department of $\mathrm{H}$ ealth and Human Services (U.S.), Substance Abuse and $M$ ental $H$ ealth Services Administration, $\mathrm{O}$ ffice of A pplied Studies. $N$ ational household survey on drug abuse. Rockville; 1999.

12 Biener L, A brams D B. The contemplation ladder: validation of a measure of readiness to consider smoking cessation. Health Psych. 1991;10(5):360-5.

13 D iClemente C, B ellino L, N eavins T. M otivation for change and alcoholism treatment. Alcohol Res H ealth. 1999;23(2):86-92.

14 Andretta I, Oliveira M S. E feitos da entrevista motivacional em jovens infratores. Estud Psicol (Campinas). 2008;25(1):45-53. 
15 M Cbride CM, Rimer BK. U sing the telephone to improve health behavior and health service delivery. Patient E duc Couns. 1999;37(1):3-18.

16 Pechansky F, Kessler F H P, D iemen L, Bumaguin D B, Sur ratt H L, Inciardi JA. Brazilian female crack users show elevated serum aluminum levels. Rev Bras Psiquiatr. 2007;29(1):39-42.

17 Duailibi LB, Ribeiro M, Laranjeira R. Perfil dos usuários de cocaína e crack no Brasil [ I nternet] . Porto Alegre: ABEAD; 2008 [ citado 2008 nov 16] . Disponível em: http:/ / www.abead.com.br/artigos/ ?cat=4.

18 Dias CA , Ribeiro M , D unn J, Sesso R, L aranjeira R. Follow-up study of crack cocaine users: situation of the patients after 2,5, and 12 years. Subst A bus. 2008;29(3):71-9.
19 Prochaska J O, D iClemente CC, N orcross JC. Search of how people change: applications to addictive behaviors. Am Psychol. 1992;47:1102-13.

20 M iller W R, Rollnick S. Entrevista motivacional: preparando as pessoas para a mudança de comportamentos adictivos. Porto Alegre: Artmed; 2001.

\section{AG RADECIMENTOS}

Os autores ag radecem à Coordenação de A perfeiçoamento Pessoal de Nível Superior (CAPE S - bolsa de pesquisa em nível de M estrado - M.C.B.), ao Consel ho $N$ acional de Desenvolvimento Científico e T ecnológico (CN Pq - bolsa de produtividade IC - H.M .T .B.) e à colaboração da Secretaria N acional de Políticas sobre D rogas (SENAD).

Recebido em: 02/ 06/ 2010

A provado em: 24/ 02/ 2011
Endereço da autora / Dirección del autor / Author's address:

Nadia K rubskaya Bisch

Rua Sarmento L eite, 245, sala 316

90050-170, Porto Alegre, RS

E-mail:nkrubs@gmail.com 